\title{
EFECTO DE ACTIVIDADES AGROPECUARIAS EN LAS CARACTERÍSTICAS DEL SUELO EN EL PÁRAMO EL GRANIZO, (CUNDINAMARCA - COLOMBIA)
}

\section{EFFECT CAUSED BY AGRICULTURAL ACTIVITIES ON SOIL CHARACTERISTICS IN THE PARAMO EL GRANIZO (CUNDINAMARCA -COLOMBIA)}

\author{
Luis Hernando Estupiñán1, Jaime Eduardo Gómez², Víctor Javier Barrantes³, Luis Fernando Limas ${ }^{4}$ \\ ${ }^{1}$ Biólogo, M.Sc. Docente Investigador, Facultad de Ingeniería Agronômica, Universidad de Ciências Aplicadas y Ambientales \\ U.D.C.A, e-mail: luishest@udca.edu.co. Dirección para correspondencia: Calle 222 No. 54-37, Bogotá, D.C. 2,3 I.A., U.D.C.A. \\ e-mail ingagro@udca.edu.co ${ }^{4}$ I.A. U.D.C.A, e-mail: llimas@hotmail.com.co
}

Rev. U.D.C.A Act. \& Div. Cient. 12 (2): 79-89, 2009

\section{RESUMEN}

Se realizó un estudio exploratorio en el páramo El Granizo (Colombia), estratégico para el Distrito Capital por su regulación hídrica y actualmente dedicado en un alto porcentaje a la agricultura y a la ganadería. El propósito de la investigación fue determinar el efecto que causan las actividades agropecuarias en algunas características físicas y químicas del suelo. Se tomaron, como referencia de comparación, zonas sin rastro de intervención antrópica, zonas con actividades agrícolas y/o pecuarias y zonas en período de descanso (barbechos). Se comprobó que las prácticas agropecuarias causan un efecto negativo sobre las propiedades del suelo del páramo. Entre las consecuencias, se destacan la compactación del suelo, el incremento de la densidad aparente y la disminución de la retención de humedad. Respecto a las propiedades químicas alteradas, se detectaron que las diversas intervenciones del hombre alteran los niveles de nitrógeno, de fósforo y la relación de bases, que influye negativamente en las condiciones naturales del páramo. Se observa, en general, que la ampliación de la frontera agrícola y pecuaria en el páramo hace que el suelo se compacte en algunos casos y se erosione en otros y que la retención de agua, su principal función, disminuya con las consecuencias negativas bien conocidas.

\section{SUMMARY}

At the paramo El Granizo (Colombia), strategic for the capital district because of its hydric regulation and actually dedicated in a high percent to agricultural activities and cattle raising, an exploratory study was performed. The purpose of the research was to determine the effect caused by these activities on some physical and chemical soil characteristics. As references, areas without traces of human actions, areas with agricultural performances and / or livestock and areas in a resting period, were evaluated. It was proven that the agricultural practices cause negative effects on the soil characteristics of the paramo. As consequences, soil compaction, increase of bulk density and the decrease of moisture retention, stand out. In reference to the altered chemical properties, it was detected that the levels of nitrogen, of phosphor and the relation of bases were altered, influencing negatively the paramo's natural conditions. In general it was observed that the augmentation of the farming frontier at the paramo makes that the soil is compacted in some cases and, in others, eroded and that the water retention, its main function, decreases, causing the well known negative consequences.

Key-words: Anthropogenic, impact, paramo.

Palabras clave: Antrópico, impacto, páramo. 


\section{INTRODUCCIÓN}

Los páramos son considerados ecosistemas estratégicos, por su alto potencial de almacenamiento y regulación hídrica. En ellos, se genera y nace gran parte de las fuentes de agua que comprende la compleja red hidrológica de una región; prestan servicios ambientales muy importantes para las comunidades rurales y urbanas (Cleef et al. 1983, Hofstede, 1995; Podwojewski E Poulenard, 2000).

Hofstede (2001) considera que los cultivos en el páramo causan el mayor impacto en el suelo, que se da desde la preparación del terreno; se elimina toda la vegetación y se voltea el suelo, éste se seca superficialmente y los nutrientes se liberan. En el primer año de cultivo, los rendimientos son relativamente altos, el cultivo utiliza las reservas de fósforo y el riesgo fitosanitario es bajo debido a la ausencia de fitopatógenos en el suelo De otra parte, los cultivos no pueden proteger al suelo de la erosión hídrica y eólica como lo hace la vegetación nativa del páramo (Laegaard, 1992).

Casanova (1991) menciona que la disparidad de valores del contenido de nutrientes en el suelo se puede relacionar con las condiciones naturales, como el relieve, el tipo de material parental y por las actividades propias de la agricultura, como el empleo de fertilizantes, de pesticidas, de especies vegetales, del manejo de residuos y, por lo general, los campesinos tratan de compensar las pérdidas de nutrientes con fertilizantes, rotando los cultivos y dejando descansar el suelo (barbechos). El problema de la fertilización de los suelos, se agrava cuando los residuos de cosechas se emplean como alimento del ganado, ya que el suelo queda al descubierto impidiendo la recarga de nutrientes, por los bajos contenidos de materia orgánica (CRQ, 2002; Lorna, 2001).

En suelos de páramo la materia orgánica presenta poco nitrógeno disponible; sin embargo, son ellos los que tienen el mayor contenido de nitrógeno. Por su parte, el potasio, junto con el magnesio, son elementos que se lixivian rápidamente en comparación con el calcio; es así que en sitios lluviosos, el contenido de potasio es bajo (Podwojewski \& Poulenard, 2000).

El pH, además de estar influenciado por la altitud y la precipitación, es afectado también por la mineralogía del suelo, siendo de débil a fuertemente ácido, cuando predomina la alófana y casi neutro cuando abunda la palagomita; en suelos con alófana es raro encontrar valores menores a cinco, debido a la capacidad amortiguadora de ésta (Sturm E Rangel, 1985).

De acuerdo con Murcia (2005), un laboreo excesivo, con mayor número de pases, actúa pulverizando los agregados del suelo, disgregándolos y deteriorando toda la estructura; esta disgregación de los agregados provoca una disminución de la red de poros, que produce una disminución de la aireación del suelo y de la capacidad de infiltración de agua de éste, que provoca, a su vez, un aumento de la escorrentía y, por tanto, de la erosión. Menciona el mismo autor que las sales de nitrato son muy solubles, posibilitando una elevada lixiviación y más si se tiene en cuenta el bajo poder de adsorción que presentan la mayoría de los suelos para las partículas cargadas negativamente; por otro lado, el problema más importante relativo al ciclo del N es la acumulación de nitratos en el subsuelo que, por lixiviación, se pueden incorporar a las aguas subterráneas o bien ser arrastrados, procedente del páramo hacia los cauces y los reservorios superficiales

Por su parte, la cría de ganado, específicamente vacuno, ha dejado una devastadora y aún no calculada pérdida de las condiciones de páramo, reflejado, especialmente, en la capacidad de retención de agua en el suelo que se ve afectada por el pisoteo constante del ganado, compactándolo y quitándole espacio poroso para acumular el agua. La pérdida de agua causa un re-acomodamiento de las unidades estructurales, produciendo modificaciones en los espacios vacíos y el suelo pierde su capacidad de infiltración, dificultando el flujo de agua y la toma de nutrientes de las plantas (Pinzón, 1989; Hofstede, 1995; CRQ, 2002). El suelo del páramo, por su alto contenido de materia orgánica, es suelto y suave, aspecto que facilita la compactación causada por el peso de los animales (Hofstede 1995). Además, las deyecciones del ganado están relacionadas con un aumento de la concentración de carbono en la capa arable y un aumento en el espesor de la capa orgánica; su mineralización presenta problemas como el exceso de nitrógeno, que afecta las características físico-químicas de los suelos y su capacidad para mantener a los cultivos, sumado los potenciales peligros de contaminación de acuíferos y cursos de agua (Murcia, 2005). 
El presente estudio buscó corroborar, para el páramo El Granizo, estratégico para el Distrito Capital y sus alrededores, por su regulación hídrica y belleza paisajística, que lo convierten en alternativa de ecoturismo para los habitantes de Bogotá y que las actividades agropecuarias deterioran las condiciones físico-químicas del suelo, causando consecuencias negativas para las funciones que desempeñan estos ecosistemas de alta montaña.

\section{MATERIALES Y MÉTODOS}

Área de estudio. Páramo El Granizo, municipio de la Calera, Cundinamarca, ubicado entre los 3.170 y 3.381m.s.n.m. Las coordenadas geográficas son $4^{\circ} 46^{\prime}$ $\mathrm{Ny} 73^{\circ} 55^{\prime} \mathrm{W}$. Presenta zonas no intervenidas y zonas con procesos de intervención, caracterizadas por las formas de ocupación antrópica, relacionadas con agricultura y con ganadería. Los sitios de muestreo, se determinaron en relación al área de la zona correspondiente, el tipo y tiempo de alteración, la variabilidad topográfica y el uso actual. Estos fueron geo-referenciados con un GPS (Global Positions System), registrando la altimetría, la latitud y la longitud. En la tabla 1, se presentan las características de cada una de las zonas.

Descripción de suelos. Se realizó a través de una calicata ubicada a una altura de 3.182m.s.n.m, representativa del área de estudio y dos cajuelas, cuyo centro se barrenó hasta encontrar impedancia. En los horizontes se determinó: textura, estructura, colorimetría, plasticidad, consistencia, presencia de niveles freáticos, actividad de organismos y máxima profundidad efectiva; presencia de materia orgánica, carbonatos y alófanas por medio de indicadores químicos, de acuerdo a las normas establecidas por la SOIL SURVEY STAFF (1998).

Características físicas y químicas del suelo: Se midió, inicialmente, la Resistencia a la Penetración (RP) a través de los diferentes horizontes, con el penetrógrafo de cono, DIK-5520, teniendo en cuenta los aspectos topográficos de cada zona, como la pendiente y la variabilidad del terreno.

Durante el estudio, se tomaron 40 muestras homogenizadas de suelo en cada una de las zonas. Se determinó: materia orgánica, nitrógeno, fósforo, potasio, capacidad de intercambio catiónico, calcio; magnesio, sodio, aluminio y saturación de bases. Los análisis, se efectuaron en el Laboratorio de Suelos del Instituto Geográfico Agustín Codazzi de Bogotá; la textura, la humedad y la densidad aparente, se analizaron en campo. Para determinar los niveles de compactación, se realizaron muestreos de la resistencia a la penetración, conjuntamente con pruebas de retención de humedad y de porosidad del suelo. La densidad aparente, se tomó

Tabla 1. Caracterización de las zonas de muestreo.

\begin{tabular}{|c|c|c|c|c|}
\hline MUESTREOS & COORDENADAS & $\begin{array}{l}\text { PENDIENTE } \\
(\%)\end{array}$ & $\begin{array}{l}\text { ALTURA } \\
\text { m.s.n.m }\end{array}$ & CARACTERÍSTICAS \\
\hline \multirow[t]{2}{*}{ M1 } & $4^{\circ} 46^{\prime} 0^{\prime \prime} \mathrm{N}$ & \multirow{2}{*}{100} & \multirow{2}{*}{3381} & \multirow{2}{*}{$\begin{array}{c}\text { Zona no intervenida. } \\
\text { Sin rastro de intervención antrópica. }\end{array}$} \\
\hline & $73^{\circ} 55^{\prime} 05^{\prime \prime} \mathrm{W}$ & & & \\
\hline \multirow[t]{2}{*}{ M2 } & $4^{\circ} 46^{\prime} 05^{\prime \prime} \mathrm{N}$ & \multirow{2}{*}{100} & \multirow{2}{*}{3326} & \multirow{2}{*}{$\begin{array}{l}\text { Zona no intervenida. } \\
\text { Sin rastro de intervención antrópica. }\end{array}$} \\
\hline & $73^{\circ} 55^{\prime} 09^{\prime \prime} \mathrm{W}$ & & & \\
\hline \multirow[t]{2}{*}{ M3 } & $4^{\circ} 46^{\prime} 11^{\prime \prime} \mathrm{N}$ & \multirow[b]{2}{*}{30} & \multirow[b]{2}{*}{3301} & \multirow{2}{*}{$\begin{array}{l}\text { Zona intervenida. } \\
\text { Diez (10) meses en descanso después de cultivo de papa } \\
\text { (Solanum tuberosum) }\end{array}$} \\
\hline & $73^{\circ} 55^{\prime} 13^{\prime \prime} \mathrm{W}$ & & & \\
\hline \multirow[t]{2}{*}{ M4 } & $4^{\circ} 46^{\prime} 51^{\prime \prime} \mathrm{N}$ & \multirow{2}{*}{15} & \multirow{2}{*}{3182} & \multirow{2}{*}{$\begin{array}{l}\text { Zona intervenida: } \\
\text { Cuarenta y ocho (48) meses en descanso después de } \\
\text { cultivo de papa (Solanum tuberosum) }\end{array}$} \\
\hline & $73^{\circ} 55^{\prime} 24^{\prime \prime} \mathrm{W}$ & & & \\
\hline M5 & $4^{\circ} 46^{\prime} 62^{\prime \prime} \mathrm{N}$ & 20 & 3170 & $\begin{array}{l}\text { Zona intervenida: Cultivo de papa con periodo vegetativo de } \\
\text { cinco meses. }\end{array}$ \\
\hline
\end{tabular}


en el Laboratorio de Suelos de la U.D.C.A, a través del método de cilindro de volumen conocido.

\section{RESULTADOS Y DISCUSIÓN}

Descripción del suelo: El horizonte superficial pertenece a un Humic Lithic Dystrudepts, relieve altamente inclinado, constituido por rocas clásticas limosas y arenosas. Suelos excesivamente drenados, marcando una profundidad de $1,60 \mathrm{~m}$, limitado por contacto rocoso; las texturas son medias a moderadamente gruesas. A la parte alta del páramo corresponde el $60 \%$ con estas características y la restante a un Andic Dystrudepts con afloramientos rocosos.

El Humic Lithic Dystrudepts se encuentra en su mayoría en la parte media área de estudio, con pendientes superiores al $75 \%$. El horizonte superficial es de color pardo grisáceo muy oscuro, textura franca, estructura en bloques subangulares, con un espesor de $25 \mathrm{~cm}$; el horizonte siguiente presenta un espesor hasta de $15 \mathrm{~cm}$ de color pardo grisáceo muy oscuro, de textura arcillosa y estructura en bloques subangulares, moderadamente, desarrollada. La clase textural de la parte alta fue arenosa-franca, lo que no permite una buena retención de humedad.

\section{Características físicas y químicas del suelo}

Resistencia a la penetración. Los valores oscilan entre 10 y $12 \mathrm{~kg} / \mathrm{cm}^{2}$, valores que se consideran aceptables para el crecimiento de las raíces; los valores por encima de $17 \mathrm{~kg} / \mathrm{cm}^{2}$ muestran una notable impedancia en el desarrollo de raíces y la presencia de organismos. Para la zona M1 (zona no intervenida), la pendiente es de $45^{\circ}$, presentándose una impedancia de $0,1 \mathrm{MPa}$, a los $10 \mathrm{~cm}$, que fue aumentando con la profundidad hasta registrarse una impedancia de $31 \mathrm{~kg} / \mathrm{cm}^{2}$, a los $80 \mathrm{~cm}$; en general, a partir de los $60 \mathrm{~cm}$ aproximadamente, existen afloramientos rocosos superficiales (Figura 1).

A partir de los $65 \mathrm{~cm}$ de profundidad, como se observa en la figura 1 , se registra una impedancia de $18 \mathrm{Kg} / \mathrm{cm}^{2}$, lo que genera problemas en el desarrollo de las raíces; sin embargo, las plantas nativas están adaptadas a estas condiciones. La textura correspondiente a esta zona es franco arenosa, lo que define una textura fina.

En la zona M3 (barbecho de diez meses), se presenta una impedancia a los $10 \mathrm{~cm}$ del horizonte superficial, con una RP de $15 \mathrm{~kg} / \mathrm{cm}^{2}$, logrando su máximo, significativamente, a los $30 \mathrm{~cm}$ con $42 \mathrm{~kg} / \mathrm{cm}^{2}$; es importante anotar que la impedancia límite para cualquier actividad agrícola, se define en los primeros $15 \mathrm{~cm}$, lo que se corrobora de acuerdo a la actividad actual de esta zona, que abarca labores de pastoreo, ocasionando el pisoteo constante sobre el horizonte superficial. Antes del pisoteo, esta zona se encontraba en cultivo de papa, cuyas labores de mecanización son excesivas utilizándose, indiscriminadamente, varios pases de arado de disco y de rotovator en los primeros $20 \mathrm{~cm}$ del horizonte, generando un pie de arado. Las actividades agropecuarias afectaron la resistencia a la penetración, RP.

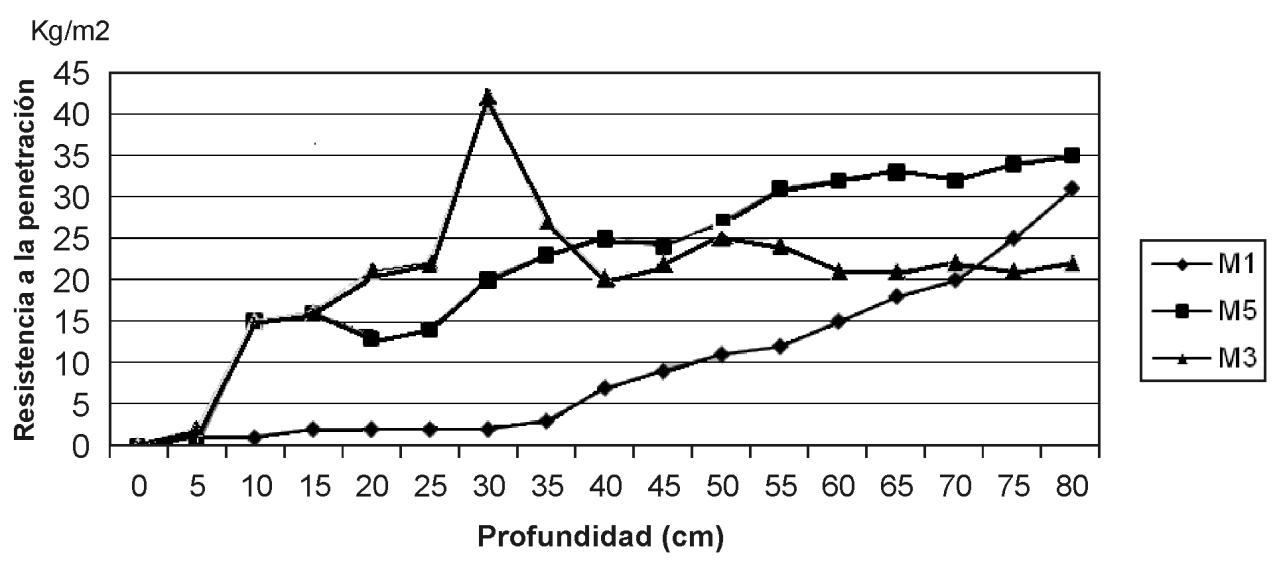

Figura 1. Comportamiento de la resistencia a la penetración en tres zonas específicas. 
Contenido de humedad en el suelo. Fue de $126,3 \%$ para el primer horizonte, en la zona M3; este valor fue de $109,6 \%$ en el mismo horizonte, por lo tanto, se aprecia que las actividades agrícolas afectaron la retención de humedad.

En la misma dinámica de la RP, se ve reflejada en la zona M5, definiendo a tan solo $10 \mathrm{~cm}$, una impedancia de $15 \mathrm{~kg} / \mathrm{cm}^{2}$; esta zona se encuentra con cultivo de papa, lo que indica una limitante para el buen desarrollo de raíces y microorganismos; esto se atribuye a la sobremecanización anterior al establecimiento del cultivo y a las prácticas culturales durante el mismo, como el caballoneo y el control de arvenses, entre otras. El establecimiento de estos cultivos en suelos con textura franco arenosa limita el mantenimiento de las condiciones naturales del ecosistema, ya que son suelos frágiles y susceptibles a cualquier tipo de alteración.

Densidad Aparente (DA). Permite definir la relación entre los espacios porosos, la retención de humedad en el suelo, la RP y el grado de actividad de organismos. A mayor DA, menor es el espacio poroso, lo que es directamente proporcional al valor de la RP en el suelo (compactación entre los horizontes), que dificulta el movimiento del agua (infiltración). Pinzón (1989), en un estudio realizado en el páramo Sumapaz, relaciona la alta retención de humedad y la baja infiltración, con el alto porcentaje de microporos. En el páramo El Granizo, la DA registrada fue en promedio, 0,63 que, en general, es considerada aceptable para el desarrollo de actividades agrícolas en cuanto a la posibilidad de crecimiento de especies vegetales y planes de fertilización, razón por la cual, se establecen cultivos en esta zona de páramo. La DA es una propiedad del desarrollo de los suelos y va cambiando a medida que va pasando el tiempo, más aun cuando se ve influenciado por actividades antrópicas, como lo muestra la figura 2 para la zona $M 3$, con un valor de $0,65 \mathrm{~g} / \mathrm{cc}$; ésta zona se encontraba, diez meses antes, con papa. Durante este periodo, se sometió a prácticas de pastoreo y de transito de maquinaria, lo que representa un aumento en comparación a la media $(0,63 \mathrm{~g} / \mathrm{cc})$ y a la del páramo propiamente dicho $(0,62 \mathrm{~g} / \mathrm{cc})$. La diferencia, se debe al tipo de suelo y al porcentaje de humedad existente $\left(0,75 \mathrm{~mm}\right.$ de agua en $\left.100 \mathrm{~cm}^{3}\right)$. Esto corrobora lo reportado por Cleef et al. (1983), en el sentido que cuando el suelo se cultiva, se seca y se compacta, no vuelve a tener su estructura original y se reduce el espacio poroso; además, en contacto con el aire, la materia orgánica se descompone, por lo que su contenido se reduce y la alófana se destruye, disminuyendo la cantidad de agua que puede retener el suelo.

La zona M4 (pastoreo semi-intensivo) registra el valor más bajo de DA, 0,61g/cc. Si bien, en la revisión de literatura, se reporta que a mayor DA menor espacios porosos, para la zona M4 no se cumple, puesto que presenta una porosidad de $1,04 \%$, lo que permite determinar que la zona está definida por su función ecológica, mas no por la actividad agrícola o pecuaria que se desarrolle (Figura 2).

En la zona $M 1$, páramo sin rastro de intervención, se registró un valor de densidad aparente de $0,62 \mathrm{~g} / \mathrm{cc}$, que se acerca al valor promedio de toda la zona de estudio y una porosidad de $1,32 \%$. En la figura 3 , se aprecia que el peso húmedo del suelo muestra valores similares en M1 y M3, que corresponden a las zonas que no sufrieron intervención o tienen periodos de descanso largos, respectivamente, mientras que para las unidades $M 4$ y M5, que estuvieron bajo actividad agrícola y pecuaria o en el caso de M5, con cultivo de papa, muestra los valores más bajos, que son directamente proporcionales a los valores del peso seco del suelo.

Se observa el efecto de las actividades agropecuarias en las características del suelo del páramo en la RP; la zona M3 refleja los valores más altos, $42 \mathrm{~kg} / \mathrm{cm}^{2}$, al igual que los valores más bajos de DA $(0,61 \mathrm{~g} / \mathrm{cc})$; mientras que en M1 los valores de DA se acercan al valor promedio del total de la zona y se registran los valores menores de RP del total de muestreos. Las actividades realizadas en la adecuación de suelos para uso agrícola ocasionaron un efecto negativo en la densidad aparente, generando una impedancia o compactación, a través de los horizontes, que afecta la infiltración del agua, necesaria para la disponibilidad de muchos nutrientes.

Es importante mencionar que los valores de densidad aparente coinciden con los reportados por Casasbuenas et al. (2006) para suelos en zonas no intervenidas del páramo Rabanal, lo que permite deducir que los incrementos en este valor podría estar relacionados con las actividades agropecuarias, que aumentaron la compactación.

Características químicas. En la figura 4, se presentan los valores de nitrógeno, fósforo y potasio. En cuanto 
A

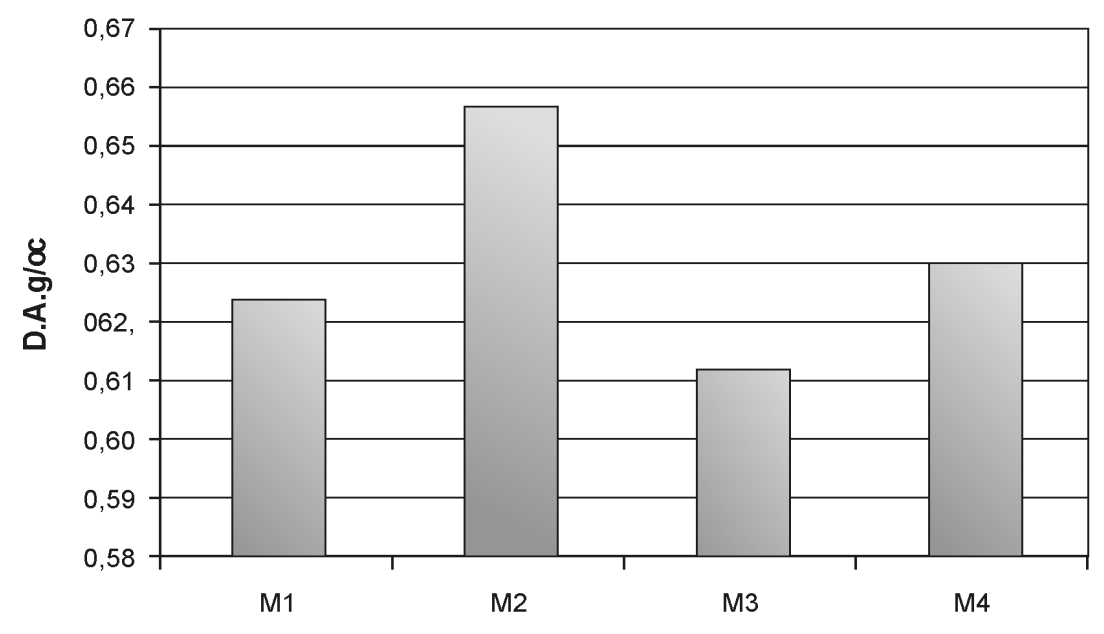

B

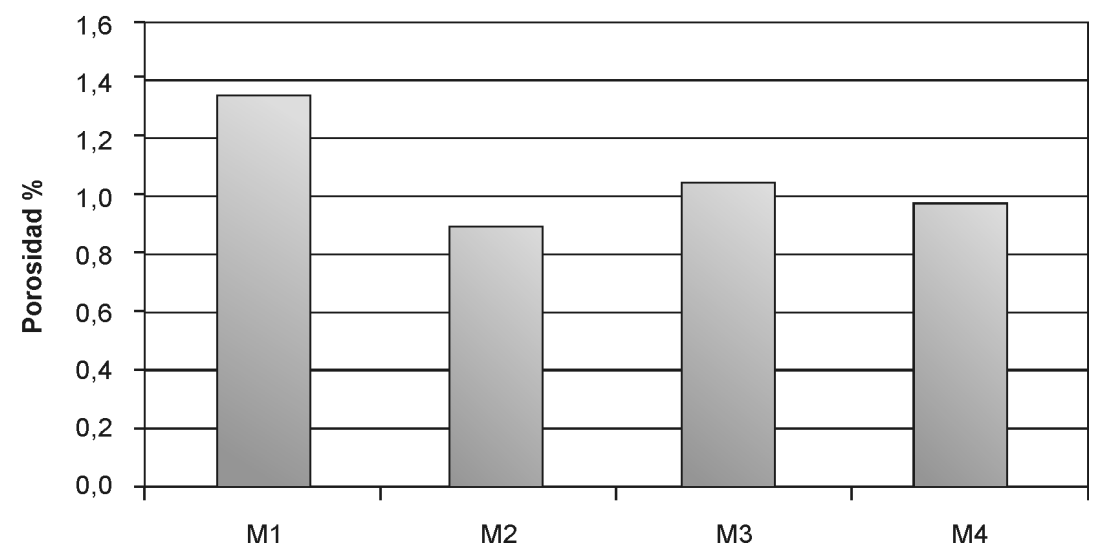

Figura 2. Variación en las diferentes zonas muestreadas. A: Densidad aparente. B: Porosidad.

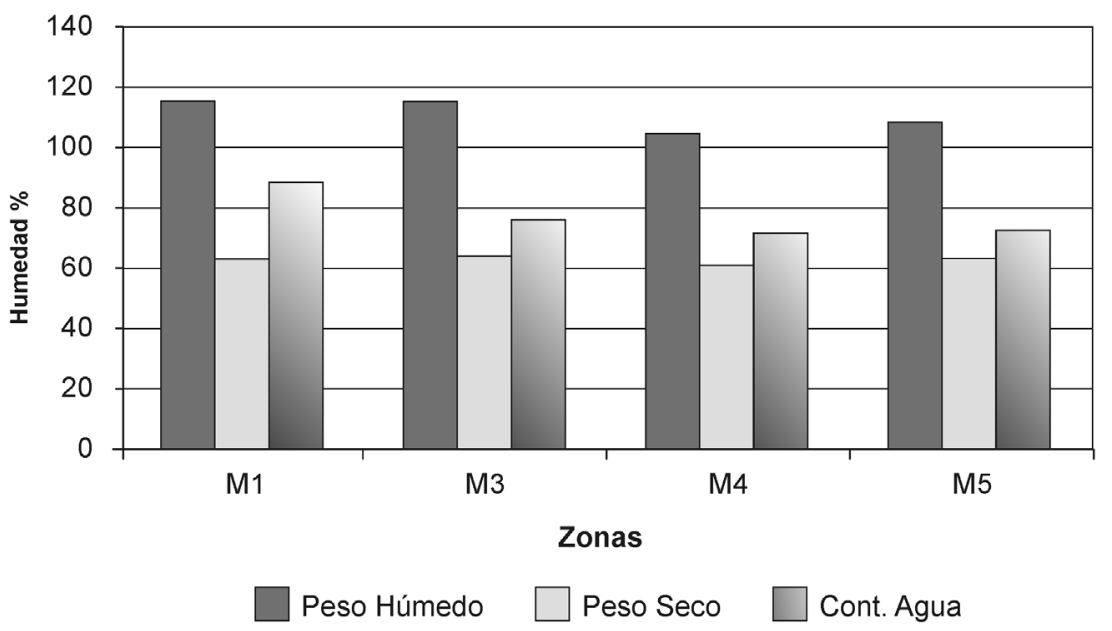

Figura 3. Relación del peso húmedo, peso seco y contenido de agua en el suelo. 
al nitrógeno, en las zonas M4 y M5, se registraron los valores más altos, 0,87 y 1,09, en época de lluvias y 1,04 y 1,02, para época seca; los mayores niveles de $\mathrm{N}$ total, se obtuvieron en la zona en cultivo de papa comparado con las demás zonas, debido a las actividades de fertilización, propias del cultivo. La principal fuente de fertilización son productos compuestos de síntesis química.

En las zonas no intervenidas y en barbechos, se halló baja cantidad de N total, posiblemente, porque se lixivia por la baja retención del suelo y por la textura franco-arenosa que predomina en la zona. Se registraron valores de N total de $0,86 \%$, para $M 1$ y $0,73 \%$, para M2. Es importante mencionar que las muestras se tomaron cuando se incrementaron las lluvias.

Los contenidos de fósforo presentes en las zonas M1 y M2 (no intervenidas), son muy bajos, si los comparamos con las zonas intervenidas (M3, M4, M5), como se muestra en la figura 4. De acuerdo a Casasbuenas et al. (2006), los valores bajos de fósforo registrados en zonas no intervenidas de páramo, se deben a los procesos de retención del ecosistema, en los que intervienen los compuestos de hierro y de aluminio y la fauna del suelo que favorecen los procesos de humificación.

El mayor contenido de fósforo es el reflejo de la adecuación de los suelos para un proceso productivo, específicamente, los valores de la zona $\mathrm{M} 3$, con un valor de 220ppm, para la época húmeda y 188ppm, para la seca, esta zona presenta un periodo de descanso de diez meses, después del cultivo de papa, por lo tanto, los niveles de fósforo no son representativos durante el periodo fenológico de la papa, sino se fijan y se disponen tiempo después. Estos resultados coinciden con los reportados para el Páramo Rabanal, por Casasbuenas et al. (2006).

En el caso de las zonas M1 y M2, los contenidos de fósforo son bajos respecto a las demás, porque estos valores se mantienen bajo condiciones naturales propias de procesos nutricionales del suelo, que tienden a estandarizarse y mantenerse constantes, durante el tiempo. Para las demás zonas, el contenido de fósforo se atribuye, en primer lugar, a la utilización de fuentes ricas de $\mathrm{P}_{2} \mathrm{O}_{5}$; en segundo, a las siembras repetitivas de hasta tres ciclos productivos seguidos, que influyen en la acumulación de este nutriente; en tercer lugar, a las condiciones del suelo, como el material parental, microorganismos, tipo de arcillas, que hacen que éste se fije y, finalmente a la poca movilidad de este elemento y la presencia de arcillas 2:1, que generan acumulaciones. Es importante mencionar que el fósforo es uno de los elementos de mayor fijación en el suelo, pero de menor disponibilidad para las plantas; por esta razón, en actividades agrícolas, se aplican fuentes con altos contenidos de este elemento.

En la zona M4, el contenido de fósforo disminuye, posiblemente, porque el periodo de descanso es mayor (48 meses). Los niveles de este nutriente bajan por la demanda nutricional de los pastos, ya que permanece en pastoreo constante. En la zona M5 (intervenida), la cual, se encuentra con cultivo de papa, el contenido de fósforo aportado por la fertilización, lo asimila en alguna proporción, la planta; por tanto, los valores son relativamente bajos en el suelo. Es importante tener en cuenta que esta zona se encontraba al final del ciclo del cultivo y se ha cumplido la mayor demanda del elemento.

Los contenidos de potasio para las zonas $M 1, M 2$, M3 y M4 no varían entre sí (Figura 4); no se muestra diferencia sobre el valor promedio en los muestreos, por las aplicaciones de fertilizantes, en comparación con el muestreo $M 5$, que registra valores entre $1,5 \mathrm{meq} / 100 \mathrm{~g}$ y $2,1 \mathrm{meq} / 100 \mathrm{~g}$, dado, posiblemente, por las excesivas aplicaciones de fertilizantes compuestos utilizados en el cultivo de papa o el empleo de quelatos, para la renovación de praderas. Estos resultados concuerdan con los reportados por Limas et al. (2002), quienes manifiestan que los niveles altos de potasio, encontrados en un estudio de barbechos, llevado a cabo en el páramo La Cumbrera, se deben a la residualidad de posibles enmiendas y de fertilización para cultivos de papa, a base de fertilizantes compuestos de N-P-K.

Los contenidos de $\mathrm{Ca}, \mathrm{Mg}$ y $\mathrm{K}$ en el suelo están relacionados con el contenido de $\mathrm{Al}$, siendo uno de los limitantes de mayor importancia ocasionadas por las actividades agrícolas y que tienen efecto directo en los contenidos de los valores de acidez en el suelo. Casasbuenas et al. (2006) explican que las relaciones bajas entre las bases de cambio en el páramo Rabanal, se debieron a la influencia del $\mathrm{pH}$ y a la saturación de aluminio. En el caso de las zonas $\mathrm{M} 1$ y M2, los valores de $\mathrm{pH}$ son muy ácidos, con un valor promedio de 3,8 (Figura 5). 

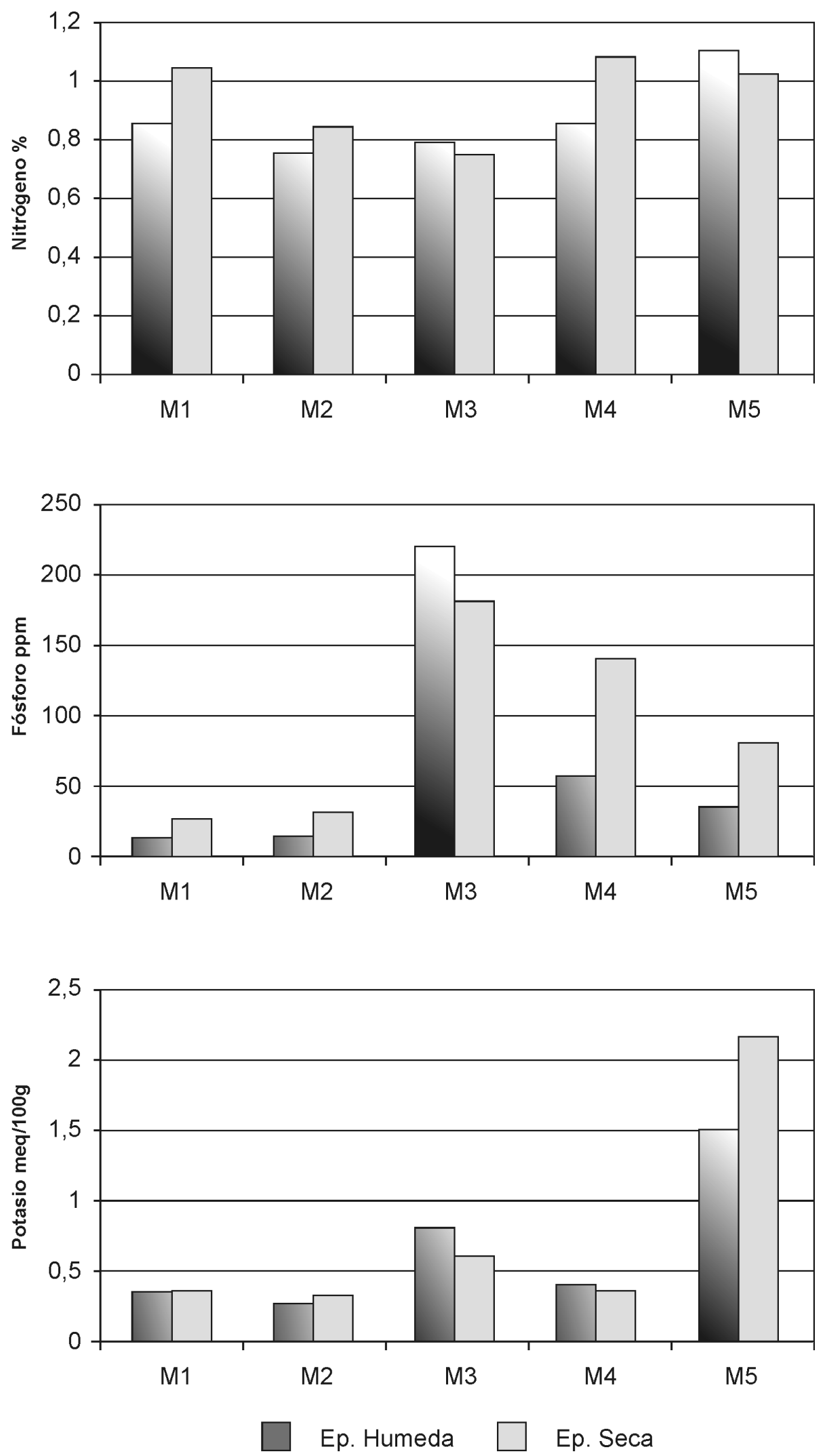

Figura 4. Contenidos de nitrógeno, fósforo y potasio en las diferentes zonas de muestreo. 
Las características de los suelos del páramo, relacionadas con contenidos de aluminio y valores de $\mathrm{pH}$ bajos, han permitido, durante mucho tiempo, la adaptabilidad de microorganismos y de especies vegetales, los cuales, reflejan las condiciones naturales de este ecosistema y, por esta condición, se hace más frágil, una vez es intervenido y perdiendo sus características naturales. De acuerdo con Moreno \& Mora-Osejo (1994), los suelos de los páramos se caracterizan por ser ácidos, como consecuencia de la presencia de minerales, como aluminio, aluminio de cambio y materia orgánica.

La relación de bases para las zonas M3, M4 y M5, bajo condiciones de humedad o sequía, aumenta con el tiempo de descanso, igualmente, cuando hay presión agropecuaria; en M3 (barbecho), la relación es alta respecto a las zonas no intervenidas. A mayor humedad la relación de bases baja y viceversa, esto se debe a que
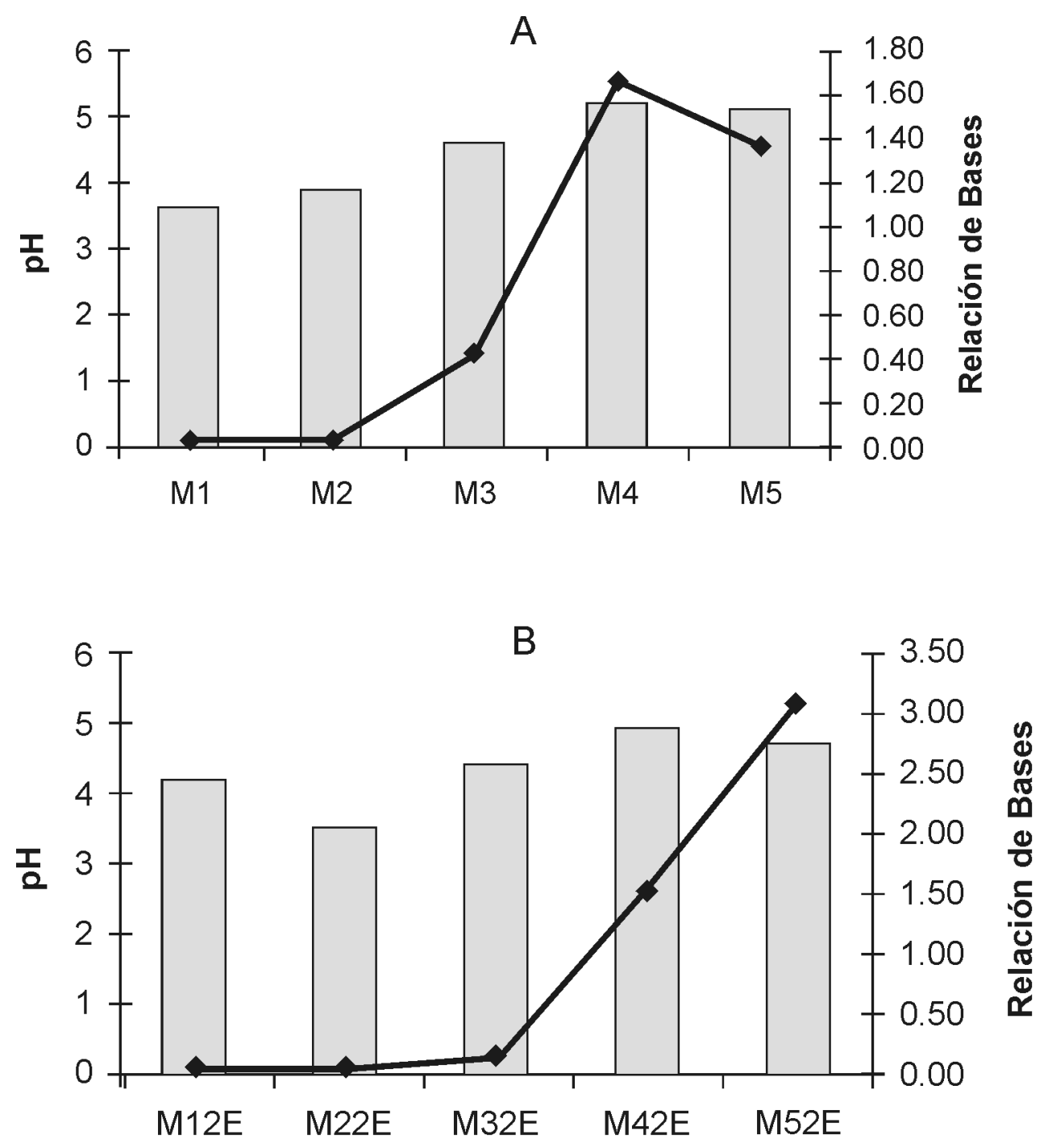

\section{$\mathrm{pH} \% \neg$ Relación de Bases}

Figura 5. Relación entre el pH y la relación de bases. A. época húmeda; B. época seca. 
el agua es un conductor de movilidad de los elementos, como el calcio en el suelo, magnesio en la planta, potasio en ambos sentidos y el aluminio es lixiviado o movilizado dentro de los horizontes, a través del perfil. Guerrero (1989, citado por Moreno \& Mora-Osejo, 1994) indica que los valores bajos de calcio, no obstante el uso de cal por los agricultores, se deben a la lixiviación que causa la deficiencia, lo mismo ocurre con el magnesio y con el potasio; además, la deficiencia de bases es una característica inherente al tipo del suelo del páramo.

En las zonas M3, M4 y M5, se reporta una relación de bases mayor a 1 alcanzando condiciones favorables para el desarrollo de cultivos; dichas condiciones son inducidas por medio de la fertilización para obtener el mayor rendimiento en las cosechas, sin importar el cambio en las propiedades químicas de la relación $\mathrm{Ca}$, $\mathrm{Mg}, \mathrm{K}, \mathrm{Al}$ y pH, lo que hace que con alta aplicación de fuentes de calcio, el pH aumente a 5,0, respecto a las zonas intervenidas. Se evidencia el efecto que tiene la adecuación de los suelos para los cultivos en el pH y la relación de bases, factores determinantes en la dinámica de los nutrimentos en el suelo del páramo.

En el proceso de acondicionamiento de los suelos de páramo para la agricultura y/o la ganadería, se aplican enmiendas, las cuales, producen un efecto en las propiedades químicas del suelo, generando cambios drásticos en la acidez y relación de bases, convirtiéndose en limitante. Una vez la vegetación de páramo es reemplazada por cultivos, el pastoreo se concentra en áreas cada vez más pequeñas y a mayor altura. El avance de la frontera agrícola y pecuaria hace que la vegetación nativa desaparezca y el suelo se erosione (Hofstede et al. 2003).

Cuando las características de los suelos de páramo presentan gran cantidad de materia orgánica, $\mathrm{pH}$ ácido, relación de bases por debajo de 1 , altos contenidos de $\mathrm{Al}$ intercambiable, niveles medios de $\mathrm{P}_{2} \mathrm{O}_{5}$ y $\mathrm{K}_{2} \mathrm{O}$ y niveles freáticos bajos, se favorecen las condiciones para que el páramo mantenga sus condiciones naturales.

Conflicto de intereses: Los autores hacen constar que el manuscrito fue preparado y revisado con la participación de todos, quienes declaramos que no existe ningún conflicto de intereses que ponga en riesgo la validez de los resultados presentados.

\section{BIBLIOGRAFÍA}

1. BARRERA, L. 2001. La fertilidad de los suelos de clima frío y la fertilización de cultivos. En: Fertilidad de suelos, diagnóstico y control. Editorial Guadalupe Ltda. Bogotá. p.419-468.

2. CASANOVA, E. 1991. Introducción a la ciencia del suelo. Consejo de Desarrollo Científico y Humanístico. U. Central de Venezuela. Caracas. 393p.

3. CASASBUENAS, L.; PRIETO, J; ESTUPIÑÁN, L.H. 2006. Efecto de la quema sobre algunas propiedades físico-químicas del suelo en el páramo Rabanal, Samacá-Boyacá. Rev. U.D.C.A Act. E Div. Cient. 9(1):97-106.

4. CLEEF, O.; RANGEL, CH.; SALAMANCA, S. 1983. Reconocimiento de la parte alta del transecto. Parque Los Nevados. En: Van Der Hammen, A.; Pérez, P.; Pinto, P. Eds. La cordillera Central Colombiana- Transecto Parque Los Nevados, Interrupción y datos iniciales. Estudios de ecosistemas tropandinos. 1:150-173.

5. CORPORACIÓN AUTÓNOMA REGIONAL DEL QUINDÍO CRQ. 2002. Cartilla Técnica del Plan de Manejo del Parque Nacional Natural Los Nevados y su Zona Amortiguadora. Cali, Colombia. 32p.

6. HOFSTEDE, R. 1995. The effects of grazing and burning on soil and plant nutrient concentrations in Colombian páramo grassland. Plant and Soil. 173(1):111-132.

7. HOFSTEDE., R. 2001. El Impacto de las Actividades Humanas sobre el Páramo. p.161-182. En: Los Páramos del Ecuador, Particularidades, Problemas y Perspectivas. Editorial Abya-Yala. Proyecto Páramo. Quito. 310p.

8. HOFSTEDE, R.; SEGARRA, P.; VASCONEZ, P. 2003. Los páramos del mundo. Global Peatland Initiative/ NC-IUCN/ Ecociencia. Quito. p.299.

9. LAEGAARD, S. 1992. Influence of fire in the grass páramo vegetation in Ecuador. En: Balsley, H.; Luteyn, J.L., eds. Páramo: Andean ecosystem under human influence. Academic Press. p.151-170. 
10. LIMAS, L.F., ESTUPIÑAN, L.H., ZENNER DE POLANÍA, I. 2002. Estudio de barbechos en el Páramo La Cumbrera y su relación con la recuperación del suelo. Rev. U.D.C.A Act. E Div. Cient. 5(1):8392.

11. LORNA, R. 2001. Factores que afectan la disponibilidad de nutrientes para las plantas. En: Siulva, F. ed. Fertilidad de suelos, diagnóstico y control. Editorial Guadalupe Ltda. Bogotá. p.29-57.

12. MORENO, O.C.; MORA-OSEJO, L.E. 1994. Estudio de los Agroecosistemas de la región de Sabaneque (Municipio de Tausa, Cundinamarca) y algunos de sus efectos sobre la vegetación. En: Mora-Osejo, L.; Sturm, H. Eds. Estudios Ecológicos del Páramo y del Bosque Altoandino. Cordillera Oriental de Colombia. Tomo II. Academia Colombiana de Ciencias Exactas, Físicas y Naturales; Colección Jorge Álvarez Lleras No. 6:89-256.

13. MURCIA C., I.; 2005. Impacto Medioambiental de las Actividades Agrícolas y Ganaderas. Modulo 2. Disponible en Internet desde: www.arrakis.es/ coag-irm/cd.htm (con acceso 02/12/06).
14. PINZÓN, P.A. 1989. Caracterización física de los suelos de los páramos Chingaza, Neusa y Sumapaz y predicción de la pérdida de suelo. Suelos Ecuatoriales. 19(1):31-40.

15. PODWOJEWSKI, P.; POULENARD, J. 2000. La degradación de los suelos de los páramos. En: Los Suelos del Páramo. Serie Páramo 5. GTP/ Abya Yala. Quito. p.27-36.

16. SOIL SURVEY STAFF. 1998. Keys to soil taxonomy. Fifth Ed. SMSS Tech, Monogr. 19. Pocahontas Press Inc. Blacksburg, Virginia. 541p.

17. STURM, H.; RANGEL, O. 1985. Ecología de los páramos andinos: Una Visión Preliminar Integrada. Instituto de Ciencias Naturales (ICN). Universidad Nacional de Colombia. Bogotá. 292p.

Recibido: Agosto 23 de 2009

Aceptado: Noviembre 9 de 2009 